\title{
ANÁLISE DOS ÍNDICES ESPECTRAIS DA VARIABILIDADE DA FREQÜÊNCIA CARDÍACA EM HOMENS DE MEIA IDADE E MULHERES NA PÓS-MENOPAUSA
}

\author{
Neves VFC ${ }^{1}$, Perpétuo NM ${ }^{2}$, Sakabe Di ${ }^{3}$, Catai AM ${ }^{1}$, Gallo Jr L ${ }^{4}$, Silva de Sá MF ${ }^{3}$, \\ Martins LEB ${ }^{5}$ E Silva E ${ }^{1,2}$ \\ ${ }^{1}$ Núcleo de Pesquisa em Exercício Físico, Departamento de Fisioterapia, Universidade Federal de São Carlos, São \\ Carlos, SP - Brasil \\ ${ }^{2}$ Laboratório de Pesquisa em Fisioterapia Cardiovascular e de Provas Funcionais, Faculdade de Ciências da Saúde, \\ Universidade Metodista de Piracicaba, Piracicaba, SP - Brasil \\ ${ }^{3}$ Departamento de Ginecologia e Obstetrícia, Faculdade de Medicina de Ribeirão Preto, Hospital das Clínicas, Universi- \\ dade de São Paulo - USP, Ribeirão Preto, SP - Brasil \\ ${ }^{4}$ Divisão de Cardiologia do Departamento de Clínica Médica, Faculdade de Medicina de Ribeirão Preto, Hospital das \\ Clínicas, USP, Ribeirão Preto, SP - Brasil \\ ${ }^{5}$ Faculdade de Educação Física, Universidade Estadual de Campinas, Campinas, SP - Brasil \\ Correspondência para: Ester da Silva, Laboratório de Pesquisa em Fisioterapia Cardiovascular e de Provas Funcionais, \\ Mestrado em Fisioterapia - Faculdade de Ciências da Saúde, Universidade Metodista de Piracicaba, Rodovia do \\ Açúcar, Km 156, CEP 13400-911, Piracicaba, SP - Brasil, e-mail: esilvas@unimep.br
}

Recebido: 29/08/2005 - Revisado: 28/03/2006 - Aceito: 26/06/2006

\begin{abstract}
RESUMO
Objetivo: Analisar e comparar a variabilidade da freqüência cardíaca (VFC), em repouso, de homens e mulheres de meia-idade. Métodos: Foram estudados 10 homens (54 \pm 3,2 anos) e 14 mulheres na pós-menopausa (56 \pm 2,6 anos) que não faziam uso de terapia hormonal. A freqüência cardíaca (FC) e os intervalos R-R foram obtidos a partir do eletrocardiograma, batimento a batimento, durante 8 minutos em repouso, nas posições supina e sentada. A VFC foi analisada no domínio da freqüência, usando a transformada rápida de Fourier, por meio da qual foram obtidas as bandas de baixa (BF) e alta freqüência (AF), as quais foram expressas em unidades normalizadas (AFun) e (BFun) e na razão BF/AF. Foram utilizados os testes estatísticos não-paramétricos de Mann-Whitney e de Wilcoxon, com nível de significância de $\alpha=5 \%$. Resultados: Na comparação intergrupo, as mulheres apresentaram maiores valores da banda AFun e menores valores da banda BFun e da razão BF/AF em relação aos homens, diferenças essas significativas $(\mathrm{p}<0,05)$. Na comparação intragrupo, não foram observadas diferenças significativas nos índices de VFC entre as posições supina e sentada para os 2 grupos estudados. Conclusão: Nossos resultados mostram uma maior modulação vagal e menor simpática no controle autonômico da FC para as mulheres em comparação aos homens de mesma idade, o que sugere que as diferenças autonômicas relacionadas ao gênero não se devem unicamente aos níveis hormonais de estrogênio, uma vez que as mulheres estudadas já se encontravam na fase pós-menopausa. Outros fatores podem estar contribuindo para essas diferenças.
\end{abstract}

Palavras-chave: variabilidade da freqüência cardíaca, homens de meia-idade, mulheres na pós-menopausa, modulação autonômica.

\section{ABSTRACT}

\section{Analysis of Spectral Indexes for Heart Rate Variability in Middle-Aged Men and Postmenopausal Women}

Objective: To analyze and compare heart rate variability (HRV) in middle-aged men and women under resting conditions. Method: Ten men (54 \pm 3.2 years) and fourteen postmenopausal women (56 \pm 2.6 years) who were not using hormonal therapy were studied. Heart rates (HR) and R-R intervals (iR-R) on a beat-to-beat basis were obtained from electrocardiograms over an eightminute period under resting conditions, in the supine and sitting positions. The HRV was analyzed in the frequency domain by means of fast Fourier transforms and the low (LF) and high (HF) frequency bands were obtained and presented as normalized 
units (LFnu and HFnu) and the LF/HF ratio. Wilcoxon and Mann-Whitney non-parametric statistical tests were used, with the significance level set at 5\%. Results: Comparing between the groups, the women presented significantly higher HFnu and lower LFnu and LF/HF ratios than did the men $(\mathrm{p}<0.05)$. Comparing within the groups, no significant differences $(\mathrm{p}>0.05)$ were found in the HRV indexes between the supine and sitting positions for either study group. Conclusion: Our results show greater vagal modulation and lower sympathetic activity in autonomic heart rate control among women than among men of similar age. This suggests that the gender-related autonomic differences are not solely dependent on estrogen levels, since the women studied were already postmenopausal. Other factors may be contributing towards these differences.

Key words: heart rate variability, middle-aged men, postmenopausal women, autonomic modulation.

\section{INTRODUÇÃO}

As mulheres, como os homens, compartilham vários fatores de risco para doença cardiovascular, tais como história familiar, dieta, obesidade, tabagismo, dislipidemia, níveis altos de homocisteína, fibrinogênio alto, sedentarismo, diabetes mellitus e hipertensão. Entretanto, as mulheres têm o fator de risco único da menopausa ${ }^{1}$. Mulheres mais jovens têm um risco significativamente menor de desenvolver doenças cardiovasculares em comparação aos homens da mesma faixa etária. No entanto, com o avanço da idade, especialmente após a menopausa, esse risco se aproxima ao dos homens ${ }^{1,2}$. Acredita-se que essa maior vulnerabilidade esteja relacionada à diminuição dos níveis hormonais de estrogênio ${ }^{3}$. Esses parecem proteger as mulheres pela promoção de um perfil lipídico antiaterogênico e pela ação direta sobre a parede dos vasos sangüíneos ${ }^{4}$.

Diversos marcadores fisiológicos são utilizados para avaliar as condições de saúde dos indivíduos, bem como estimar o risco de acometimentos cardiovasculares através de reflexos gerais e específicos. O sistema nervoso autônomo exerce grande efeito modulador sobre o sistema cardiovascular em condições fisiológicas e patológicas. Arcos reflexos aferentes enviam informações a partir do sistema nervoso central e periférico para os centros autonômicos cerebrais no bulbo, sendo as mesmas processadas e enviadas de volta ao coração por vias eferentes. As respostas desenvolvemse de forma muito rápida e têm como finalidade básica manter a homeostase local.

A variabilidade da freqüência cardíaca (VFC) é uma importante ferramenta de avaliação não-invasiva da integridade da função neurocardíaca. Ela reflete a interação das eferências simpática e parassimpática sobre o nodo sinusal. No entanto, para se avaliar a relativa contribuição de cada uma dessas eferências na modulação autonômica cardíaca, são utilizadas análises no domínio da freqüência 5 .

Estudos prévios têm relatado importantes alterações do controle autonômico da freqüência cardíaca (FC) em função da idade ${ }^{6,7}$ e do nível de condicionamento físico ${ }^{8,9}$, em indivíduos de ambos os sexos. Na mulher, a redução da VFC decorrente do envelhecimento parece estar associada à depressão dos níveis hormonais de estrogênio que ocorre em razão da menopausa ${ }^{7,10}$. Diferenças autonômicas relacionadas ao gênero também têm sido reportadas na literatura. A maior dominância vagal sobre a regulação da FC observada nas mulheres pode ser responsável pela cardioproteção dessas comparadas aos homens ${ }^{6,11}$. No entanto, com o envelhecimento, essas diferenças tendem a desaparecer ${ }^{6}$.

Considerando-se que, após a menopausa, tem sido observado um aumento na incidência de doenças cardiovasculares ${ }^{1,2}$ e que reduções nos índices de VFC tem sido associados a um aumento no risco de ocorrências de eventos cardiovasculares ${ }^{12}$, torna-se necessário que estudos sejam realizados, avaliando índices que refletem a integridade cardiovascular, tanto no homem como na mulher pós-menopausa, uma vez que tais informações são relevantes tanto para a avaliação como para a intervenção precoce. Diante disso, o objetivo do presente estudo foi analisar e comparar a VFC de homens de meia-idade e de mulheres na pós-menopausa, saudáveis e sedentários, em condições de repouso, nas posições supina e sentada.

\section{MATERIAIS E MÉTODOS}

Este trabalho foi aprovado pelo Comitê de Ética em Pesquisa da instituição (parecer $n^{\circ}$ 019/2001). Todos os voluntários foram informados e esclarecidos a respeito do protocolo experimental a que seriam submetidos e assinaram um termo de consentimento formal, aceitando participar do estudo.

Para inclusão dos voluntários, neste trabalho, foram considerados os seguintes critérios: estilo de vida sedentário; não tabagistas; não usuários de drogas que causassem dependência química; não obesos; não portadores de anormalidades do sistema cardiovascular, respiratório ou musculoesquelético; não usuários de medicações anti-hipertensivas, cardioativas e antivertiginosas. Para o grupo de mulheres na pós-menopausa também foram consideradas a ausência de fluxo menstrual espontâneo há mais de 12 meses consecutivos e a concentração sérica do hormônio folículo estimulante (FSH) maior do que $30 \mathrm{mIU} / \mathrm{ml}$.

Foram estudados 24 voluntários saudáveis, sendo 14 mulheres na fase pós-menopausa que não faziam uso de terapia hormonal e 10 homens de meia-idade. A idade e as características antropométricas dos grupos estudados estão apresentadas na Tabela 1. 
Tabela 1. Idade, características antropométricas e clínicas dos grupos estudados.

\begin{tabular}{ccc}
\hline Variáveis & Mulheres (n=14) & Homens (n=10) \\
\hline Idade (anos) & $56 \pm 2,6$ & $54 \pm 3,2$ \\
Estatura (cm) & $158 \pm 6,1$ & $167 \pm 5,2^{\mathrm{a}}$ \\
Massa corporal $(\mathrm{kg})$ & $62 \pm 9,4$ & $73 \pm 8,9^{\mathrm{a}}$ \\
IMC $\left(\mathrm{kg} / \mathrm{m}^{2}\right)$ & $24,8 \pm 3,2$ & $26,1 \pm 2,6$ \\
FC (bpm) & $67 \pm 7,5$ & $65 \pm 7,4$ \\
PAS (mmHg) & $118 \pm 17,6$ & $118 \pm 6,3$ \\
PAD (mmHg) & $76 \pm 10,5$ & $79 \pm 3,4$
\end{tabular}

Dados expressos em média \pm DP. IMC = índice de massa corporal; FC = freqüência cardíaca; PAS = pressão arterial sistólica; PAD pressão arterial diastólica; ${ }^{a} \mathrm{p}<0,05$ em relação às mulheres pósmenopausa.

Todos os voluntários apresentaram resultados dos exames laboratoriais (hemograma, glicemia de jejum, colesterol total e frações, triglicérides e urina rotina) e do eletrocardiograma em repouso dentro dos limites de normalidade; o resultado do teste ergométrico foi negativo para isquemia miocárdica e arritmias em todos os casos. Nesse teste, os voluntários foram classificados quanto a sua capacidade aeróbia e todos foram considerados como "fracos”, de acordo com a American Heart Association.

O estudo foi realizado em laboratório climatizado artificialmente, com temperatura ambiente mantida em torno de $23^{\circ} \mathrm{C}$ e a umidade relativa do ar em torno de $50 \%$. Foi promovida a familiarização progressiva de cada voluntário com os equipamentos, pessoal, local, avaliação e testes que foram aplicados.

Foi orientado a todos os voluntários que não ingerissem bebidas alcoólicas e/ou estimulantes (chá, café e outros) 24 horas antes dos testes, que tivessem uma boa noite de sono, que não utilizassem medicamentos e que fizessem uma refeição leve pelo menos 2 horas antes do teste.

Antes da realização do protocolo experimental, foi realizado um repouso inicial por um período de 15 minutos, na posição supina, para que as variáveis cardiovasculares, pressão arterial (PA) e FC retornassem aos valores basais.

O eletrocardiograma (ECG) e a FC foram captados batimento a batimento, em tempo real, por meio de uma interface entre um monitor cardíaco de um canal (TC500, ECAFIX, São Paulo, SP, Brasil) e um microcomputador (Pentium III 500MHz). O sinal analógico do ECG, captado a partir da derivação MC5 (pólo negativo no manúbrio esternal, pólo positivo posicionado no quinto espaço intercostal, na linha hemiclavicular esquerda, e o neutro no quinto espaço intercostal direito), foi digitalizado com uma freqüência de amostragem de $500 \mathrm{~Hz}$ por meio de um conversor analógicodigital (Lab. PC+, NATIONAL INSTRUMENTS, CO, Austin, TX, USA) e processado por um software específico (STEF 1.01 PC-LPM-16 PnP) ${ }^{13}$, que realizava o cálculo dos intervalos R-R e da FC em valores instantâneos, amostrados em tempo real e armazenados em disco, permitindo, assim, a análise posterior dos dados.

Os voluntários foram orientados a se manterem em repouso, a respirarem espontaneamente e a não conversarem com os experimentadores. Após os 15 minutos iniciais de repouso, iniciou-se a coleta da FC durante 8 minutos em cada posição: supina e sentada.

A análise VFC foi realizada no domínio da freqüência. Para isso, utilizou-se o procedimento de retirada de tendência linear, e a transformada rápida de Fourier foi aplicada em janela única, na seqüência dos valores dos intervalos R-R, com o mínimo de 256 batimentos consecutivos ${ }^{5}$, e foram computadas as potências espectrais em três bandas de: muito baixa freqüência (MBF: < 0,04 Hz), de baixa freqüência (BF: 0,04$0,15 \mathrm{~Hz}$ ) e de alta freqüência (AF: 0,15-0,4 Hz). Neste estudo, utilizamos as bandas de BF e AF, que melhor representam a atuação dos componentes simpático e vagal no controle da FC, respectivamente ${ }^{5}$. Esses componentes espectrais foram expressos em unidades normalizadas (BFun e AFun), as quais correspondem ao percentual do espectro total de potência subtraído do componente de $\mathrm{MBF}^{5}$. Tais componentes ainda foram expressos como a razão entre eles (razão BF/AF), indicativa do balanço simpato-vagal ${ }^{5}$.

\section{Análise Estatística}

A idade, as características antropométricas e clínicas foram analisadas pelo teste $t$ de student e apresentadas de forma descritiva, em média e desvio-padrão. Foi aplicado o teste de Kolmogorov-Smirnov para avaliar a distribuição de normalidade dos valores dos índices espectrais de VFC. Pela observação de não-normalidade na distribuição dos conjuntos de dados analisados, foram aplicados os testes nãoparamétricos de Wilcoxon e Mann-Whitney. Foi considerado o nível de significância de $\alpha=5 \%$. Os resultados foram apresentados graficamente em Box-plots, contendo os valores da mediana, primeiro (25\%) e terceiro (75\%) quartis, valores máximos e mínimos, outliers e extremos.

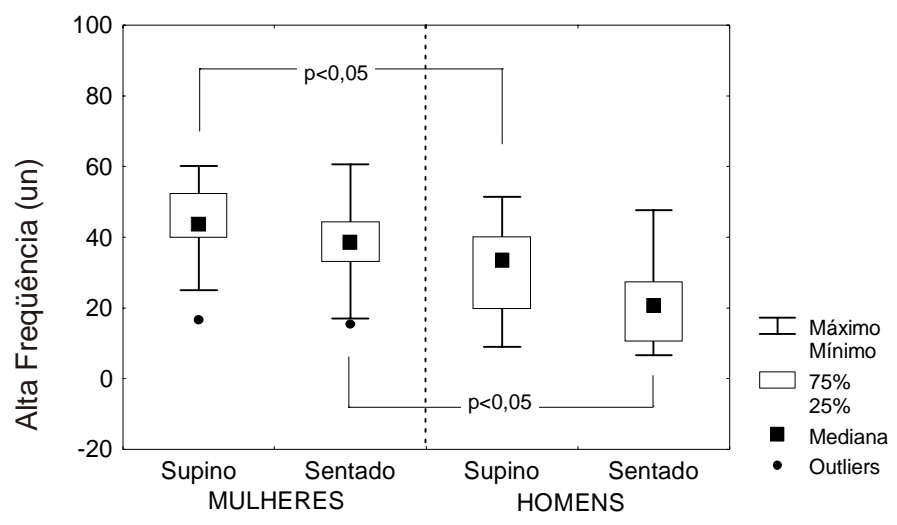

Figura 1. Valores do componente de alta freqüência, em unidades normalizadas (un), dos voluntários estudados nas condições de repouso supino e sentado. 


\section{DISCUSSÃO}

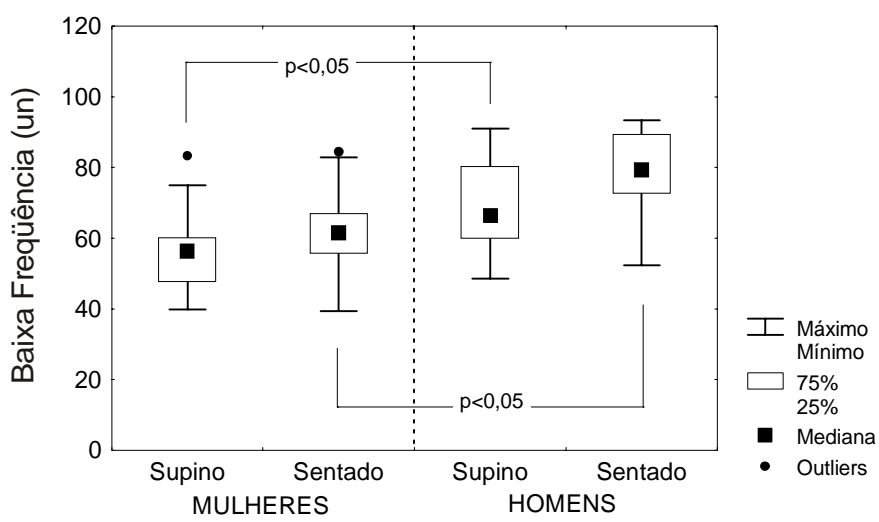

Figura 2. Valores do componente de baixa freqüência, em unidades normalizadas (un), dos voluntários estudados nas condições de repouso supino e sentado.

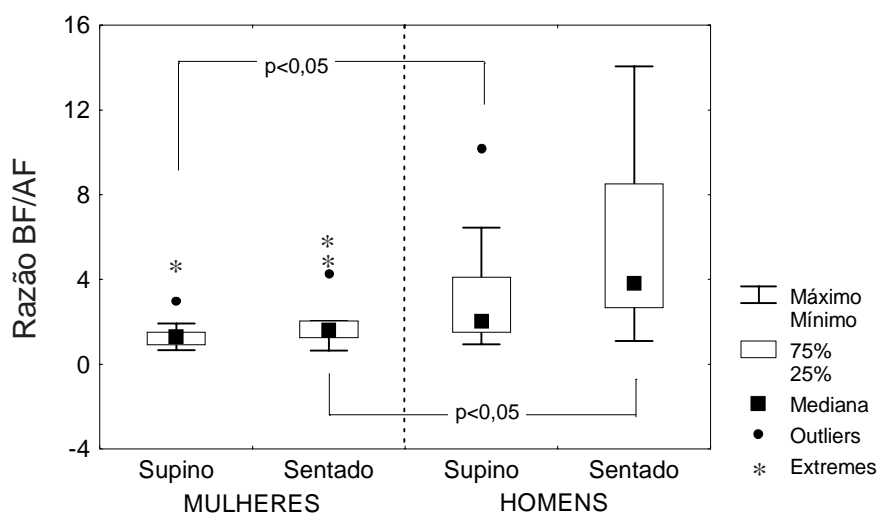

Figura 3. Valores da razão entre os componentes de baixa e alta freqüência (razão BF/AF) dos voluntários estudados nas condições de repouso supino e sentado.

\section{RESULTADOS}

Não foram observadas diferenças significativas $(p>0,05)$ entre os grupos quanto à idade e ao índice de massa corporal (Tabela 1). As mulheres pós-menopausa apresentaram valores inferiores de estatura e de massa corporal em relação aos homens $(\mathrm{p}<0,05)$. Quanto à $\mathrm{FC}$ e à $\mathrm{PA}$, em repouso, foram encontrados resultados similares entre os grupos estudados $(\mathrm{p}>0,05)$.

Na comparação intergrupo dos índices espectrais de VFC (Figuras 1 a 3 ), as mulheres pós-menopausa apresentaram valores superiores do componente de AFun e inferiores do componente de BFun e da razão BF/AF em relação aos homens $(\mathrm{p}<0,05)$, tanto na posição supina como na posição sentada. Na comparação intragrupo, não foram observadas diferenças significativas dos índices espectrais de VFC, entre as posições supina e sentada, para os 2 grupos estudados $(\mathrm{p}>0,05)$.
A análise da VFC é uma técnica simples, não invasiva e sensível de avaliação da modulação do sistema nervoso autônomo sobre o coração e tem sido utilizada para avaliar diferentes populações, como as estudadas no presente estudo.

Está bem estabelecido na literatura que o controle autonômico da FC declina progressivamente com o avanço da idade. Esse processo tem sido demonstrado pela redução tanto dos índices que refletem a atividade vagal como daqueles que refletem a atividade simpática sobre o coração $0^{6,14}$. No entanto, os mecanismos responsáveis para essa resposta fisiológica não são completamente conhecidos. Podem estar relacionados às alterações estruturais e funcionais no miocárdio e vasos sangüíneos, à sensibilidade dos tecidos, à atividade baro-reflexa, entre outros ${ }^{15}$.

Estudos prévios têm reportado diferenças na modulação autonômica cardíaca entre homens e mulheres. A maioria desses estudos mostra que as mulheres possuem uma maior atividade vagal e uma menor atividade simpática quando comparada aos homens ${ }^{6,16,17}$, e que essas diferenças entre os gêneros diminuem com o envelhecimento ${ }^{6,18}$.

No entanto, a mulher apresenta um fator diferencial no processo fisiológico do envelhecimento, que é o advento da menopausa. Devido à maior incidência de doenças cardiovasculares em homens comparados às mulheres até a idade da menopausa, pressupõe-se que a menopausa constituiu um significante marco cardiovascular em termos de fisiologia e patologia².

Alguns autores têm observado que o estrógeno exerce um efeito protetor no sistema cardiovascular da mulher ${ }^{4,11}$ e, com o advento da menopausa, a redução em sua produção acarreta um aumento na atividade simpática, evidenciado pela análise da VFC, o qual, por sua vez, é revertido com o uso crônico de terapia estrogênica ${ }^{10,19}$.

No presente estudo, foi observado que as mulheres apresentaram valores superiores da banda de AFun e inferiores da banda de BFun e da razão BF/AF em relação aos homens, o que reflete uma maior atividade vagal e uma menor atividade simpática atuante sobre o nodo sinoatrial nas mulheres estudadas $^{5}$. Esses resultados indicam que as diferenças relacionadas ao gênero, na regulação autonômica cardíaca, ainda estão presentes nessa faixa etária. Considerando que as mulheres do presente estudo se encontram na fase após a menopausa e que as mesmas não são usuárias de hormônios, isso sugere que outros fatores, e não apenas os níveis fisiológicos de estrogênio, podem contribuir para tais diferenças. Desde que a banda da BF é um componente que reflete as oscilações do sistema baro-reflexo ${ }^{5}$, nossos achados também podem estar indicando diferenças na função baroreceptora entre homens e mulheres.

Nossos resultados corroboram os achados de outros autores que também observaram valores inferiores dos índices espectrais que refletem a atividade simpática ${ }^{17,20}$ e superiores 
daqueles que refletem a atividade vagal ${ }^{17,20,21}$ sobre o controle da FC nas mulheres em comparação aos homens de meiaidade. Ryan et al. ${ }^{22}$ observaram maiores valores da razão AF/ BF em mulheres jovens, de meia-idade e idosas, em relação aos homens de mesma idade, sugerindo uma maior participação vagal sobre o controle da FC. Ressalta-se que a forma de análise desse autor ${ }^{22}$, difere da forma proposta pelo Task Force ${ }^{5}$, que propõe a normatização da análise no domínio da freqüência. Assim, tem sido proposto que a razão seja calculada pela BF/AF. No estudo de Jensen-Urstad et al. ${ }^{14}$ foram observados maiores valores da banda de BF e da razão BF/ AF para os homens em todas as faixas etárias estudadas. Sakabe et al. ${ }^{23}$ não encontraram diferenças dos índices temporais da VFC entre mulheres na pós-menopausa e homens de meia-idade.

No entanto, Liu et al. ${ }^{11}$ verificaram menores valores da banda de AF e maiores valores da razão BF/AF nos homens de meia-idade em relação às mulheres pré e pós-menopausa, em uso de terapia estrogênica; mas nenhuma diferença foi observada entre os homens e as mulheres pós-menopausa não usuárias de hormônios. No estudo de Kuo et al. ${ }^{6}$, as diferenças entre homens e mulheres nos índices espectrais de VFC desapareceram a partir da idade de 50 anos. Ramaekers et al. ${ }^{18}$ observaram valores significativamente superiores da banda de BF nos homens em comparação às mulheres, apenas nos indivíduos mais jovens (idade $<39$ anos).

Quanto à posição corporal, a literatura refere que durante a mudança postural de supino para sentado, ocorrem ajustes cardiovasculares em decorrência de a força de gravidade agir contrariando o retorno venoso para o coração, resultando num volume sistólico reduzido e FC aumentada para a manutenção do débito cardíaco. Também têm sido observadas variações no tônus vago-simpático, envolvendo a ativação das vias eferentes simpáticas e menor ativação vagal para o coração ${ }^{24}$.

No presente estudo, não foram observadas diferenças significativas nos valores de VFC entre as posições supina e sentada nos 2 grupos estudados. Embora estudos prévios conduzidos em indivíduos jovens ${ }^{25}$ e de meia-idade ${ }^{8,17}$ tenham mostrado alterações nos índices de VFC com a mudança postural, outros autores ${ }^{23,26}$ verificaram que, com o avanço da idade, tais alterações perdem sua significância estatística. Isso pode ser atribuído às modificações estruturais arteriais e às suas propriedades mecânicas decorrentes do envelhecimento, as quais parecem ser o evento primário no desencadeamento das alterações cardíacas. Assim, o aumento do diâmetro e da espessura das paredes arteriais, causado pela deposição de cálcio e por modificações na natureza do colágeno e da elastina na camada média vascular, leva à redução da elasticidade e a um aumento da rigidez das paredes das mesmas $^{27}$. O predomínio dos mecanismos constritores, mediados por diversas substâncias vasoativas, estimula a hipertrofia e a hiperplasia da musculatura lisa; o aumento da matriz extracelular, com conseqüente redução da complacência, e o aumento da resistência vascular na elevação da pressão arterial ${ }^{28}$.

Nossos resultados mostram uma maior modulação vagal e menor simpática no controle autonômico da FC para as mulheres em comparação aos homens de mesma idade, o que sugere que as diferenças autonômicas relacionadas ao gênero não se devem unicamente aos níveis hormonais de estrogênio, uma vez que as mulheres estudadas já se encontravam na fase pós-menopausa. Outros fatores podem estar contribuindo para a menor atividade simpática sobre o coração das mulheres. Se essas diferenças podem protegê-las contra o desenvolvimento de arritmias e de doenças cardíacas, requer estudos futuros, com um desenho longitudinal.

Agradecimentos: Os autores são gratos à Júlio César Crescêncio e Renata Torres Kozuki, pela assistência durante a realização do protocolo experimental. Apoio: CNPq ( $\left.{ }^{\circ} 140684 / 2003-4\right)$, FAPESP $\left(n^{\circ}\right.$ 01/074272) e FAP-UNIMEP.

\section{REFERÊNCIAS BIBLIOGRÁFICAS}

1. Genazzani AR. Controversial issues in climacteric medicine (I) Cardiovascular disease and hormone replacement therapy. Maturitas. 2001; 38(3): 263-71.

2. Hayward CS, Kelly RP, Collins P. The roles of gender, the menopause and hormone replacement on cardiovascular function. Cardiovasc Res. 2000; 46(1): 28-49.

3. Greendale GA, Lee NP, Arriola ER. The menopause. Lancet. 1999; 353(9152): 571-80.

4. Mendelsohn ME. Protective effects of estrogen on the cardiovascular system. Am J Cardiol. 2002; 89(12 Suppl 1): S12-7.

5. Task Force of the European Society of Cardiology and the North American Society of Pacing and Electrophysiology. Heart rate variability: standards of measurement, physiological interpretation and clinical use. Circulation. 1996; 93(5): 1043-65.

6. Kuo TBJ, Lin T, Yang CCH, Li CL, Chen CF, Chou P. Effect of aging on gender differences in neural control of heart rate. Am J Physiol. 1999; 277(6 Pt 2): 2233-9.

7. Ribeiro TF, Azevedo GD, Crescêncio JC, Marães VRFS, Papa V, Catai AM, et al. Heart rate variability under resting conditions in postmenopausal and young women. Braz J Med Biol Res. 2001; 34(7): 871-7.

8. Davy KP, Miniclier NL, Taylor JA, Stevenson ET, Seals DR. Elevated heart rate variability in physically active postmenopausal women: a cardioprotective effect? Am J Physiol. 1996; 271(2 Pt 2): 455-60.

9. Melo RC, Santos MDB, Silva E, Quitério RJ, Moreno MA, Reis MS, et al. Effects of age and physical activity on the autonomic control of heart rate in healthy men. Braz J Med Biol Res. 2005; 38(9): 1331-8.

10. Mercuro G, Podda A, Pitzalis L, Zoncu S, Mascia M, Melis $\mathrm{GB}$, et al. Evidence of a role of endogenous estrogen in the modulation of autonomic nervous system. Am J Cardiol. 2000; 85(6): 787-9.

11. Liu CC, Kuo TB, Yang CCH. Effects of estrogen on gender-related autonomic differences in humans. Am J Physiol. 2003; 285(5): 188-93. 
12. Bigger JT, Fleiss JL, Steinman RC, Rolnitzky LM, Kleiger RE, Rottman JN. Frequency domain measures of heart period variability and mortality after myocardial infarction. Circulation. 1992; 85(1): 164-71.

13. Silva E, Catai AM, Trevelin LC, Guimarães JO, Silva Jr LP, Silva LMP, et al. Design of a computerized system to evaluate the cardiac function during dynamic exercise [abstract]. Phys Med Biol. 1994; 39a: 409.

14. Jensen-Urstad K, Storck N, Bouvier F, Ericson M, Lindblad LE, Jensen-Urstad M. Heart rate variability in healthy subjects is related to age and gender. Acta Physiol Scand. 1997; 160(3): 235-41.

15. Walsh RA. Cardiovascular effects of the aging process. Am J Med. 1987; 82 Suppl 1B: S34-40.

16. Evans JM, Ziegler MG, Patwardhan AR, Ott JB, Kim CS, Leonelli FM, et al. Gender differences in autonomic cardiovascular regulation: spectral, hormonal, and hemodynamic indexes. J Appl Physiol. 2001; 91(6): 2611-8.

17. Pikkujämsä SM, Mäkikallio TH, Airaksinen KEJ, Huikuri HV. Determinants and interindividual variation of R-R interval dynamics in healthy middle-aged subjects. Am J Physiol. 2001; 280(3): 1400-6.

18. Ramaekers D, Ector H, Aubert AE, Rubens A, Van de Werf F. Heart rate variability and heart rate in healthy volunteers. Is the female autonomic nervous system cardioprotective? Eur Heart J. 1998; 19(9): 1334-41.

19. Rosano GMC, Patrizi R, Leonardo F, Ponikowski P, Collins P, Sarrel PM, et al. Effect of estrogen replacement therapy on heart rate variability and heart rate in healthy postmenopausal women. Am J Cardiol. 1997; 80(6): 815-17.

20. Huikuri HV, Pikkujämsä SM, Airaksinen KEJ, Ikäheimo MJ, Rantala AO, Kauma H, et al. Sex-related differences in autonomic modulation of heart rate in middle-aged subjects. Circulation. 1996; 94(2): 122-5.
21. Bigger JT Jr, Fleiss JL, Steinman RC, Rolnitzky LM, Schneider WJ, Stein PK. RR variability in healthy, middle-aged persons compared with patients with chronic coronary heart disease or recent acute myocardial infarction. Circulation. 1995; 91(7): 1936-43.

22. Ryan SM, Goldberger AL, Pincus SM, Mietus J, Lipsitz LA. Gender and age-related differences in heart rate dynamics: are women more complex than men? J Am Coll Cardiol. 1994; 24(7): 1700-7.

23. Sakabe DI, Catai AM, Neves VFC, Oliveira L, Silva de Sá MF, Azevedo GD, et al. Análise da modulação autonômica do coração durante condições de repouso em homens de meia idade e mulheres pós menopausa. Rev Bras Fisioter. 2004; 8(1): 89-95.

24. Lindqvist A. Noninvasive methods to study autonomic nervous control of circulation. Acta Physiol Scand. 1990;588 Suppl 1: S107.

25. Low PA, Opfer-Gehrking TL, Proper CJ, Zimmerman I. The effect of aging on cardiac autonomic and postganglionic sudomotor function. Muscle Nerve. 1990;13(2): 152-7.

26. Perini R, Veicsteinas A. Heart rate variability and autonomic activity at rest and during exercise in various physiological conditions. Eur J Appl Physiol. 2003; 90(3-4): 317-25.

27. Lakatta EG, Levy D. Arterial and cardiac aging: major shareholders in cardiovascular disease enterprises. Circulation. 2003;107(1 Pt 1): 139-46.

28. Lüscher TF, Dohi Y, Tschudi M. Endothelium-dependent regulation of resistance arteries: alterations with aging and hypertension. J Cardiovasc Pharmacol. 1992; 19 Suppl 5: S34-42. 